\title{
An Evolutionary Approach for Competency-based Curriculum Sequencing
}

\author{
Luis de-Marcos, José-Javier Martínez, José-Antonio Gutiérrez, Roberto Barchino \\ José-María Gutiérrez \\ Computer Science Department, Polytechnic School \\ University of Alcala. Spain \\ +34918856651 \\ \{luis.demarcos, josej.martinez, jantonio.gutierrez, roberto.barchino, josem.gutierrez\}@uah.es
}

\begin{abstract}
The process of creating e-learning contents using reusable learning objects (LOs) can be broken down in two sub-processes: LOs finding and LO sequencing. Sequencing is usually performed by instructors, who create courses targeting generic profiles rather than personalized materials. This paper proposes an evolutionary approach to automate this latter problem while, simultaneously, encourages reusability and interoperability by promoting standards employment. A model that enables automated curriculum sequencing is proposed. By means of interoperable competency records and LO metadata, the sequencing problem is turned into a constraint satisfaction problem. Particle Swarm Optimization (PSO) and Genetic Algorithm (GA) agents are designed, built and tested in real and simulated scenarios. Results show both approaches succeed in all test cases, and that they handle reasonably computational complexity inherent to this problem, but PSO approach outperforms GA.
\end{abstract}

\section{Categories and Subject Descriptors}

K.3.1 [Computers and Education] Computer Uses in Education Computer-assisted instruction (CAI).

\section{General Terms}

Experimentation

\section{Keywords}

e-Learning, Learning Object Sequencing, Competency, Particle Swarm Optimization (PSO), Genetic Algorithm (GA)

\section{INTRODUCTION}

Curriculum sequencing is a technique used to build adaptive learning resources. Currently, adaptive hypermedia approaches offer [2] the most usual solution to solve this problem but these methods yield proprietary systems and non-portable courseware. On the other hand, the LO paradigm encourages [6] reusability and interoperability but it is assumed that it clearly hinders adaptivity. We propose a standard-based adaptive framework for intelligent LO sequencing in which reusable competency definitions [5] were attached to LOs' metadata in order to define restrictions between two LOs. By defining a competency (or a set of competencies) as a LO outcome, and by defining the same competency as the prerequisite for another LO, a constraint between the two LOs is established so that the first one must precede the second LO in a valid sequence. Given a random LOs'

Copyright is held by the author/owner(s).

GECCO'08, July 12-16, 2008, Atlanta, Georgia, USA.

ACM 978-1-60558-130-9/08/07. sequence modeled in this way, the issue of finding a correct sequence can be modeled as a permutation constraint satisfaction problem (permutCSP). The solution space comprises all possible sequences ( $n$ ! will be its size for $n$ LOs), and a (feasible) solution is a sequence that satisfies all established constraints.

Two different evolutionary computation approaches were used to build several agents that solve the sequencing problem: A discrete fully-informed particle swarm optimization (PSO) agent [3]; and a genetic algorithm (GA) agent with order recombination, swap mutation and generational replacement with elitism ( $k$-size tournament selection and a duplicate elimination policy were also implemented). Fitness function for both agents was a standard penalty function.

\section{EXPERIMENTAL RESULTS}

Both algorithms for were designed and implemented using the object oriented paradigm. We wanted to test their performance in real and simulated scenarios. As a real-world problem, we choose a problem concerning course sequencing for a Master in Engineering (M.Eng.) program in our institution. The (web engineering) M.Eng, program comprises 23 courses (subjects) grouped in:

- Basic courses (7) that must be taken before any other (kind of course). There may be restrictions between two basic courses, for example 'HTML' course must precede Javascript course,

- 'Itinerary' courses (5) that must be taken in a fixed ordered sequence.

- Compulsory courses (5). There may be restrictions between two compulsory courses.

- Elective courses (6). Additional constraints with respect to any other course may be set.

All courses have an expected learning time that ranges from 30 to 50 hours. They are delivered online using a LMS, namely EDVI LMS [1], and every course has its metadata record. Competency records were created to specify LOs' restrictions, and LOM [4] metadata records were updated to reflect prerequisite and learning outcome competencies. A feasible sequence must have 23 LOs satisfying all constraints. The graph showing all LOs and constraints is very complex, and so it is to calculate the exact number of feasible solutions. Some estimation have been used, we have estimated that the relation among feasible solutions and total solutions order is $8,9 \times 10^{12}$. This number reflects the number of states (non-feasible solutions) for each feasible solution. One hundred tests were run computing mean fitness values evolution 
using the best configuration found for each agent (Figure 1). Both agents converge, but PSO approach outperforms GA.

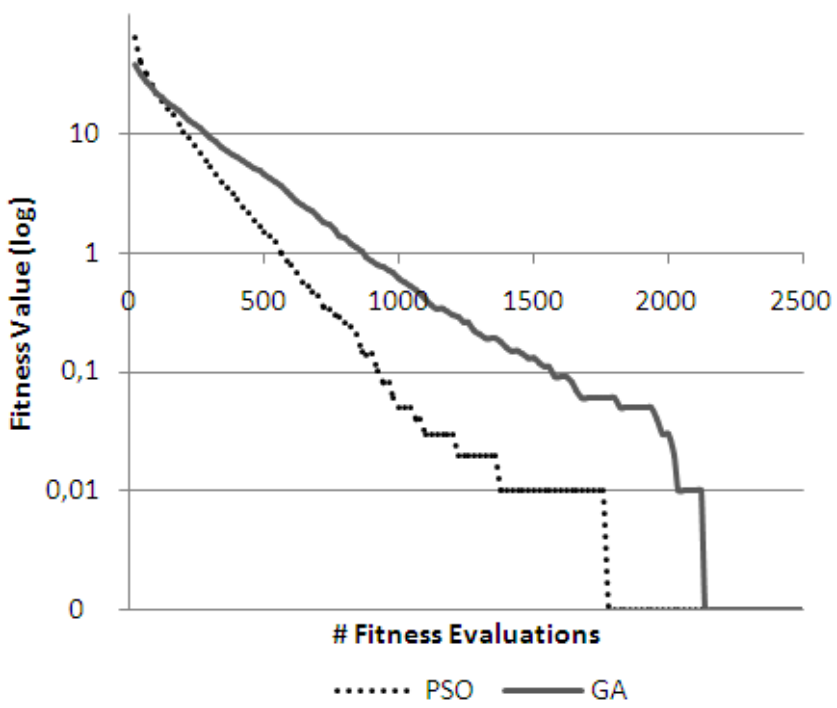

Figure 1. Agents performance in a real world problem.

The tested scenario may seem to have many feasible solutions that would make doubtful PSO performance in more 'challenging' scenarios, so additional test were conducted. Test sequences ranging from 5 to 100 LOs with only one feasible solution were designed. Each test suite was run 100 times for each agent and mean values were computed. Figure 2 shows the results and it also supports the argument that PSO outperforms GA. It could also be inferred that both agents handle reasonably combinatorial explosion for this particular problem.

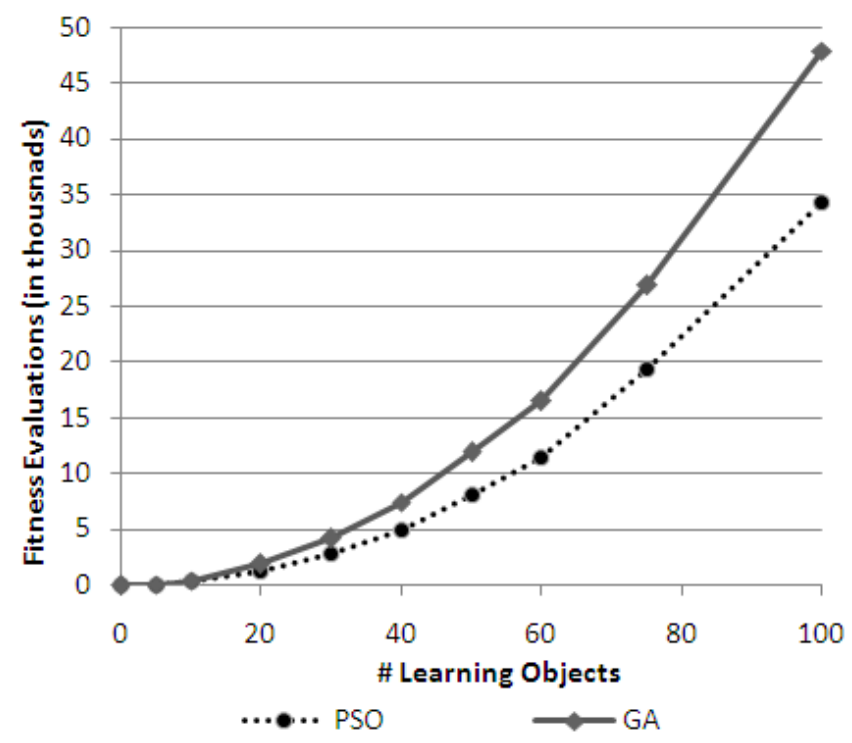

Figure 2. Agents scalability in simulated scenarios

\section{CONCLUSIONS}

Automated LO sequencing is a recurring problem in the elearning field that could be approached employing models that ensure interoperability along with artificial intelligent techniques. The purpose of the study was to design, develop and test two agents that perform automatic LO sequencing through competencies in order to study its completeness and performance. A model that employs competencies as a mean for defining constraints between learning object has been presented, so that a sequence of LOs is represented by relations among LOs and competencies. New sequences can be derived if permutation operations are allowed between LOs in the sequence. Hence the sequencing problem is turned into a permutation problem, and the aim is to find a sequence that satisfies all restrictions expressed in the original model. A GA that handles permutation problems has been developed and the PSO for permutation problem has been extended to LO sequencing problem. Results show that both agents succeed in solving the problem and that PSO implementation outperforms GA agent.

Further implications arise from the model proposal: (1) E-learning standards are promoted. XML records and bindings are used, so elements will be easily interchanged and processed by compliant systems. (2) Instructor's role is automated reducing costs. Sequencing process works even in complex scenarios were humans face difficulties. Instructors could spend saved time performing other activities within the learning action. And (3), the model can be extended to an automated intelligent system for building personalized e-learning experiences. Sequencing process can be complemented with gap analysis process and competency learner modeling techniques to build personalized courses. These courses could also be standards compliant, so they could be imported to current systems.

\section{ACKNOWLEDGMENTS}

This research is co-funded by: (1) the University of Alcalá FPI research staff education program, (2) MITyC PROFIT program (grants FIT-350200-2007-6 and FIT-350101-2007-9) and Plan Avanza program (grant PAV-070000-2007-103), (3) the MEC PROFIT program (grant CIT-410000-2007-5) and (4) Castilla-La Mancha autonomous community educational innovation cooperation program (grant EM2007-004).

\section{REFERENCES}

[1] Barchino, R., Gutiérrez, J.M. and Otón, S., An Example of Learning Management System. in IADIS Virtual Multi Conference on Computer Science and Information Systems (MCCSIS 2005), (Virtual, 2005), IADIS Press, 140-141.

[2] Brusilovsky, P. Adaptive and Intelligent Technologies for Web-based Education. Künstliche Intelligenz, Special Issue on Intelligent Systems and Teleteaching, 4. 19-25.

[3] de-Marcos, L., Pages, C., Martinez, J.J. and Gutierrez, J.A., Competency-Based Learning Object Sequencing Using Particle Swarms. in 19th IEEE International Conference on Tools with Artificial Intelligence. ICTAI, (Patras, Greece, 2007), IEEE Press, 111-116.

[4] IEEE. Learning Technology Standards Committee (LTSC). Learning Object Metadata (LOM). 1484.12.1, IEEE, 2002.

[5] IMS. Reusable Definition of Competency or Educational Objective - Information Model, IMS Global Learning Consortium, 2002.

[6] Wiley, D.A. Connecting learning objects to instructional design theory: A definition, a metaphor, and a taxonomy. in Wiley, D.A. ed. The Instructional Use of Learning Objects, 2000 . 